\title{
Correction to: Sighting of southern right whale dolphin (Lissodelphis peronii) in the Magellan Strait, Chile
}

\author{
Marco Pinto-Torres ${ }^{1,2} \cdot$ Jorge Acevedo $^{3,4} \cdot$ Carla Mora $^{5} \cdot$ Eva Iglesias $^{2} \cdot$ Diego Bravo-Gómez $^{2} \cdot$ Francisco Martínez ${ }^{6}$
}

Published online: 19 March 2019

○) Springer-Verlag GmbH Germany, part of Springer Nature 2019

\section{Correction to: Polar Biology (2019) 42:633-638 https://doi.org/10.1007/s00300-018-02446-4}

This correction stands to correct the co-author name originally credited as: Diego Bravo. The author group would like readers to know that the name should be credited as Diego Bravo-Gómez and not the former. The original article has been corrected.

Publisher's Note Springer Nature remains neutral with regard to jurisdictional claims in published maps and institutional affiliations.

The original article can be found online at https://doi.org/10.1007/ s00300-018-02446-4.

Jorge Acevedo

jorge.acevedo@cequa.cl; jorge.acevedo@live.cl

1 Programa de Doctorado en Ciencias de La Acuicultura, Universidad Austral de Chile, Los Pinos S/N, Puerto Montt, Chile

2 Centro FONDAP de Investigación de Ecosistemas Marinos de Altas Latitudes (IDEAL), Universidad Austral de Chile, Av. El Bosque 01789, Punta Arenas, Chile

3 Centro de Estudios del Cuaternario de Fuego-Patagonia y Antártica (Fundación CEQUA), Avenida España 184, Punta Arenas, Chile

4 Programa de Doctorado en Ciencias Marinas y Costeras, Universidad Autónoma de Baja California Sur, Carretera al Sur Km 5.5, 23080 La Paz, BCS, Mexico

5 Independent Researcher, Carlos Condell 0290, Punta Arenas, Chile

6 Fitz Roy Expeditions S.A., Roca 825, Punta Arenas, Chile 